\title{
Structure and Morphology Effects on the Optical Properties of Bimetallic Nanoparticle Films Laser Deposited on a Glass Substrate
}

\author{
A. O. Kucherik, ${ }^{1}$ S. M. Arakelyan, ${ }^{1}$ S. V. Kutrovskaya, ${ }^{1}$ A. V. Osipov, ${ }^{1}$ \\ A. V. Istratov, ${ }^{1}$ T. A. Vartanyan, ${ }^{2}$ and T. E. Itina ${ }^{3}$ \\ ${ }^{1}$ Stoletov's Vladimir State University (VSU), 87 Gorki Str., Vladimir 600000, Russia \\ ${ }^{2}$ ITMO University, 49 Kronverksky Pr., Saint Petersburg 197101, Russia \\ ${ }^{3}$ Université de Lyon, UJM-Saint-Etienne, CNRS, UMR5516, Laboratoire Hubert Curien, 42023 Saint-Etienne, France \\ Correspondence should be addressed to T. E. Itina; tatiana.itina@univ-st-etienne.fr
}

Received 27 November 2016; Revised 6 February 2017; Accepted 20 April 2017; Published 9 May 2017

Academic Editor: Mohamed Bououdina

Copyright (C) 2017 A. O. Kucherik et al. This is an open access article distributed under the Creative Commons Attribution License, which permits unrestricted use, distribution, and reproduction in any medium, provided the original work is properly cited.

Moving nanosecond laser system is used for laser-assisted thermodiffusion deposition of metallic nanoparticles from water-based colloidal solutions. The results obtained for both gold and silver nanoparticles show that film morphology strongly depends on laser scanning speed and the number of passages. We show, furthermore, the possibility of producing bimetallic Au:Ag thin films by laser irradiation of the mixed solutions. As a result of several laser scans, granular nanometric films are found to grow with a well-controlled composition, thickness, and morphology. By changing laser scanning parameters, film morphology can be varied from island structures to quasi-periodic arrays. The optical properties of the deposited structures are found to depend on the film composition, thickness, and mean separation between the particles. The transparency spectra of the deposited films are shown to be defined by their morphology.

\section{Introduction}

Efficient and well-controlled synthesis of thin films with desired optical properties is a key issue in modern photonics. In particular, possibilities of plasmon-assisted light manipulations by using nanoparticles and nanostructures currently attract considerable attention enabling various applications. Laser-assisted deposition of nanostructured metal films on a glass substrate is one of the promising formation methods for these structures. In fact, this method is rather easy.

In particular, the optical properties of nanoparticle films are known to be dominated by a set of plasmonic effects depending not only on the film composition but also on its morphology and periodicity [1]. Such parameters as spacing and ordering of nanoparticles define the local field enhancement in the case of nanoparticle structures $[2,3]$. If nanoparticle spacing is smaller or comparable with their sizes, the optical properties of the ordered structures differ from these of the random ones [4-6]. These structures, however, have also a great potential for many applications, since the ordered deposition of the individual particles is rather time-consuming and difficult to scale up [7]. Therefore, new synthesis methods of random structures are under development and currently attract particular interest of the researchers working in various fields ranging from photonics to medicine and biology. One of such new methods was recently developed $[8,9]$ enabling not only an efficient deposition of metallic nanoparticles on glass substrates, but also a control over their optical properties by changing the thickness of thin films and spacing between the particles.

In this work, we perform laser-assisted deposition of bimetallic Au:Ag nanoparticles from colloidal solutions. In the proposed method, colloidal nanoparticles are deposited on glass by using local laser heating leading to the particle movement toward the substrate [9]. Based on our previous studies $[8,9]$, here we present the results of much more detailed investigations focused not only on the influence of 


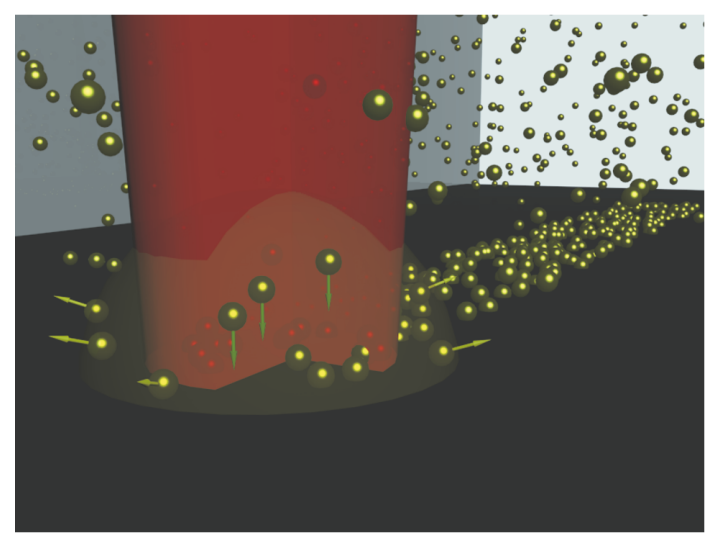

FIGURE 1: Schematics of laser-induced thermodiffusion deposition of colloidal nanoparticles.

such parameters as the distance between the deposited particles and the number of the deposited layers, but also on the film deposition process and, in particular, on the connections with the resulting morphology and optical properties of the films. We believe that the results of this much more systematic study provide a more complete understanding of both laserassisted film formation processes and of their properties. Herein, the optical properties of the obtained films are then investigated both experimentally and numerically. In addition to the previously used calculations performed based on the solution of Maxwell's equations [9], here we also present the results of a direct simulation of the deposition process, as well as of the generalized Mie simulations aimed at the elucidation of the film morphology roles. In particular, we focus attention here on the possibility of using fractal nanoparticle aggregates and structures in order to change film optical parameters. The ways of an efficient control over the optical properties of the obtained nanoparticle structures are, thus, proposed.

\section{Deposition of Bimetallic Thin Films}

2.1. Laser-Assisted Deposition of Metallic Colloidal Nanoparticles. The initial colloidal systems were prepared by continuous wave $(\mathrm{CW})$ laser ablation of silver and gold targets in deionized water. The mean power of $\mathrm{CW}$ laser used here was $30 \mathrm{~W}$, spot diameter was $100 \mu \mathrm{m}$, and spot velocity was $100 \mu \mathrm{m} / \mathrm{s}$. The average particle sizes were determined by the dynamic light scattering technique yielding $\sim 10 \mathrm{~nm}$, while their concentration was $\sim 1 \mathrm{mg} / \mathrm{ml}$. More details about nanoparticle formation by $\mathrm{CW}$ laser in liquids can be also found in $[10,11]$.

For nanoparticle deposition from the obtained solutions, Ytterbium fiber laser was then used with wavelength of $1.06 \mu \mathrm{m}$, pulse duration of $100 \mathrm{~ns}$, repetition rate of $20 \mathrm{kHz}$, and laser pulse energy up to $1 \mathrm{~mJ}$. The diameter of the laser beam at the focal plane was $5 \mu \mathrm{m}$. Figure 1 shows the schematics of the experimental procedure. Here, a KV8 glass substrate was placed in a cuvette with the nanoparticle solution. Typically, nanoparticle deposition was proceeded by scanning the laser beam along the same direction from 5 to
25 times. The scanning speed was varied from $0.6 \mathrm{~mm} / \mathrm{s}$ to $1.5 \mathrm{~mm} / \mathrm{s}$.

Our previous estimations have shown that nanoparticle temperature in the colloids reaches $\sim 600 \mathrm{~K}$ for a single laser passage [12]. With an increase in the number of laser passages, heat accumulation typically takes place leading to the laserinduced convection and thermal diffusion ensuring nanoparticle motion toward the substrate surface. The diffusion coefficient of spherical nanoparticles in water is determined by using Einstein's equation as follows:

$$
D_{0}=\mu k T,
$$

where $\mu$ is particle mobility, $k$ is the Boltzmann constant, and $T$ is liquid temperature. For spherical particles, Stokes mobility $\mu=(6 \pi v r)^{-1}$, where $v$ is the kinematic viscosity of the liquid and $r$ is particle radius. When the mixture is heated to $600^{\circ} \mathrm{K}$, the estimated diffusion coefficient $D \sim$ $10^{-10} \mathrm{~cm}^{2} \mathrm{~s}^{-1}$ for nanoparticles with $r=10 \mathrm{~nm}$ in water. We note that this value corresponds only to the diffusion coefficient in the laser-heated volume, whereas $D$ is as small as $10^{-18} \mathrm{~cm}^{2} \mathrm{~s}^{-1}$ close of the substrate surface [12].

The proposed deposition method allows one to change the morphology of the deposited structures depending on the laser exposure conditions. Figure 2 displays two typical AFM images of the deposited colloidal systems consisting only of silver or gold nanoparticles.

The deposited structures are composed of easily distinguishable discrete particles with dimensions from 10 to $50 \mathrm{~nm}$. A detailed analysis of the AFM images confirms the formation of agglomerates during particle deposition process, where a fraction of the nanoparticles start moving toward the substrate upon laser heating [10]. With the increase in the number of laser passages, island structure is formed.

The nanoparticle deposition process is modeled as follows. The simulated rectangular volume with learned dimensions of $10 \mu \mathrm{m}$ is subdivided into $200 \times 200 \times 200$ cubic cells. Then, randomly moving nanoparticles are introduced into 50 cells located at the bottom in a cylinder with the radius of 10 cells, where laser heating is simulated. The laser heating is modeled by changing the diffusion coefficient from 0 to 1 . Upon reaching the bottom boundary of the model area, the particle is considered to be deposited. The obtained results are shown in Figure 3 that represents quite well the obtained experimental results.

\subsection{Laser-Assisted Formation of the Bimetallic Au:Ag Granular} Coatings. For bimetallic films, an equal amount of silver and gold colloids of equal concentration was mixed. Then, depending on the number of laser passages, the formation of bimetallic Au:Ag films proceeded as a set of several consecutive stages as a function of the number of laser scans.

At the beginning, island films $[8,9,13]$ are found to be formed. This result is typically obtained after as few as 5 laser passages at a scanning speed of $1.2 \mathrm{~mm} / \mathrm{s}$ (Figure $4(\mathrm{a})$ ). The average height of the relief obtained at this stage is $14 \mathrm{~nm}$. After 10 scans (Figure 4(b)), the islands become significantly larger, and the average height of the relief reaches $18 \mathrm{~nm}$. 


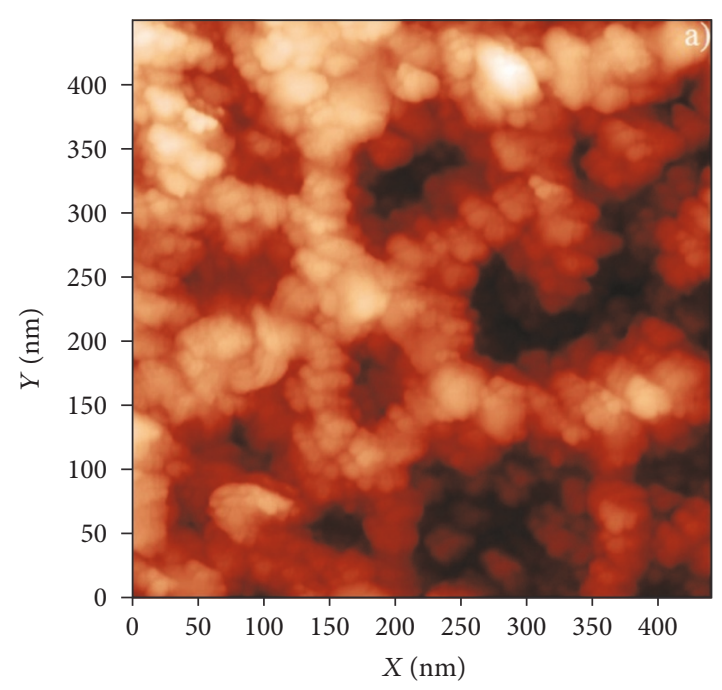

(a)

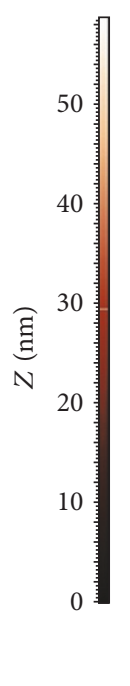

Figure 2: AFM images of gold (a) and silver (b) particle deposited on the substrate. Here, laser power is $2.5 \mathrm{~W}$; the structures are formed for 25 laser passages with the scanning speed of $1.2 \mathrm{~mm} / \mathrm{s}$.

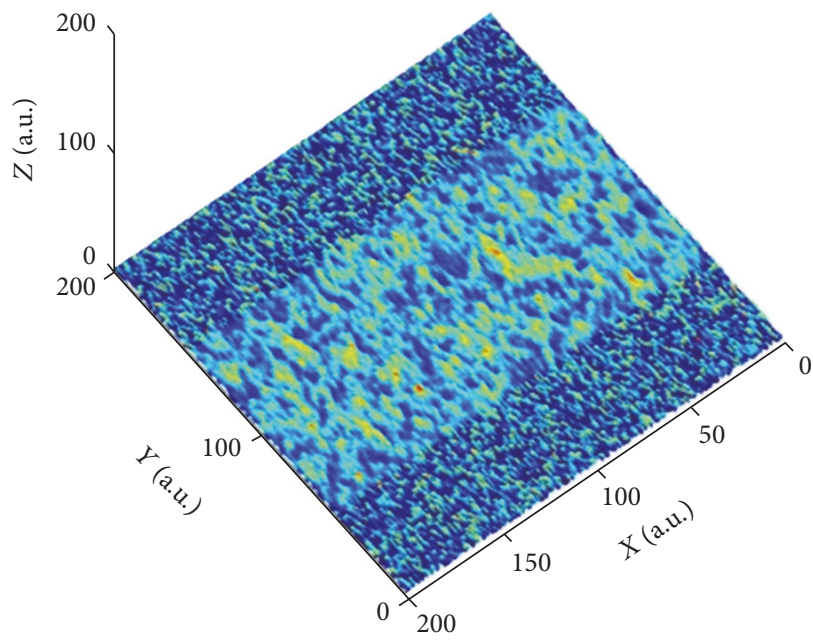

(a)

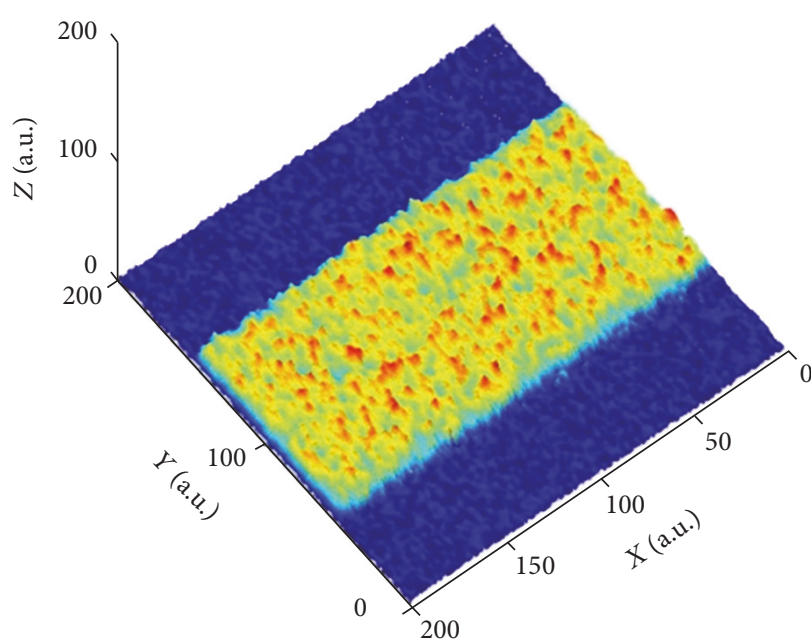

(c)

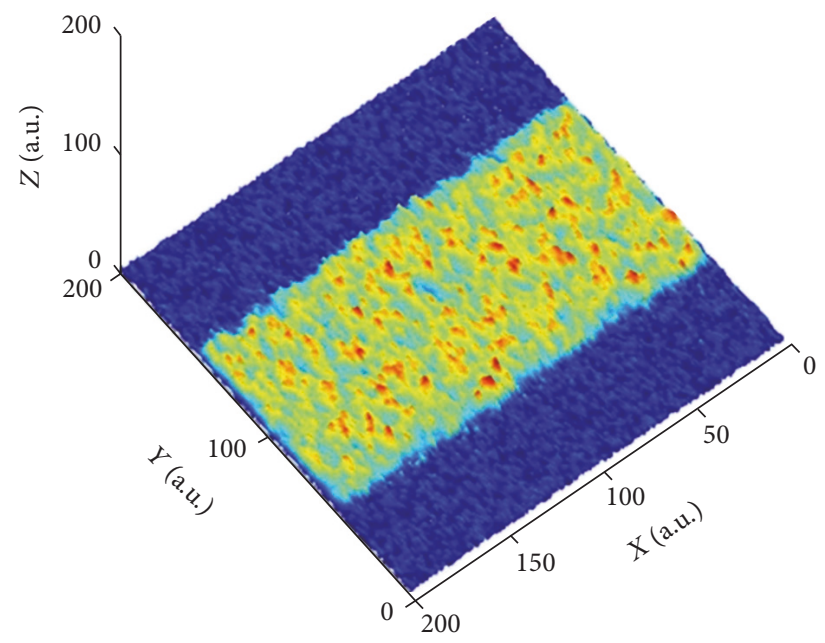

(b)

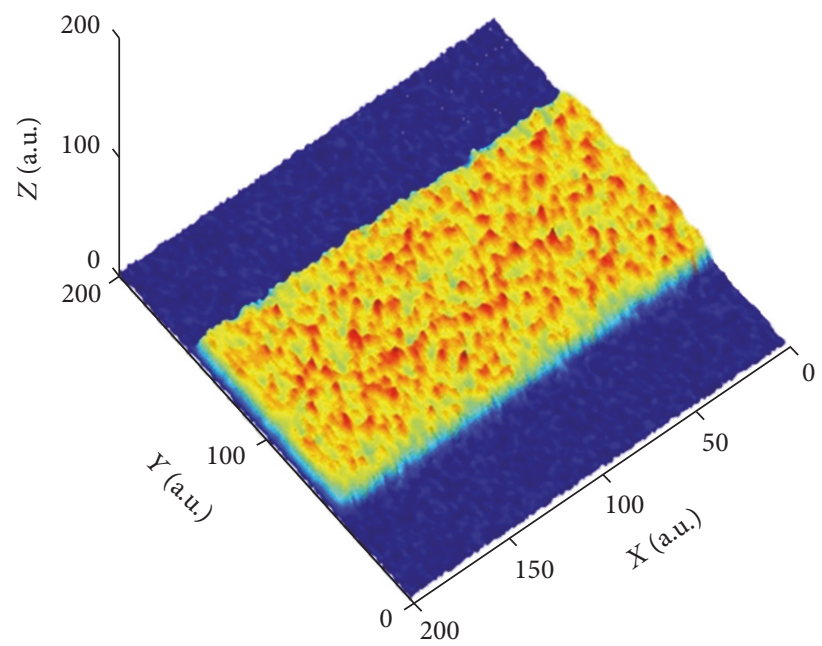

(d)

FiguRE 3: Simulation results obtained for the deposition process after 5 (a), 10 (b), 15 (c), and 20 (d) scans of laser beam. 


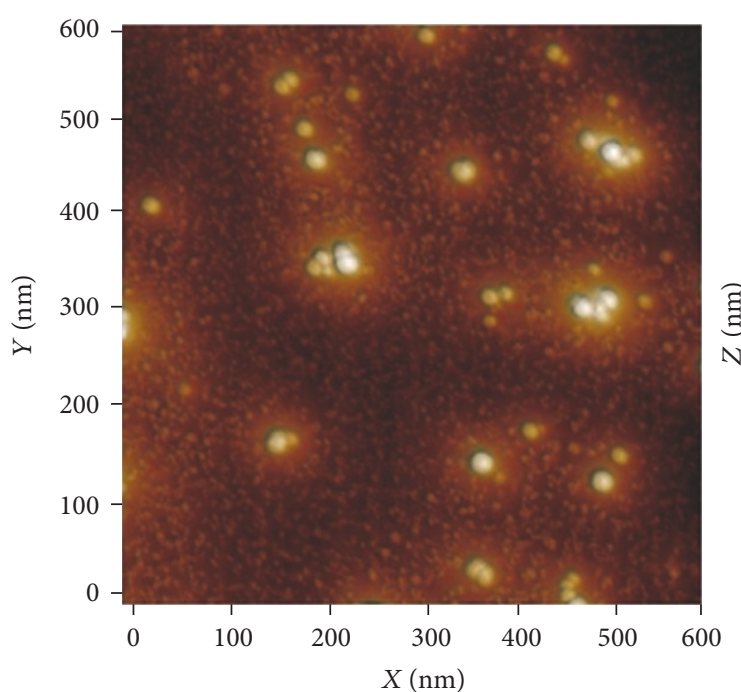

(a)

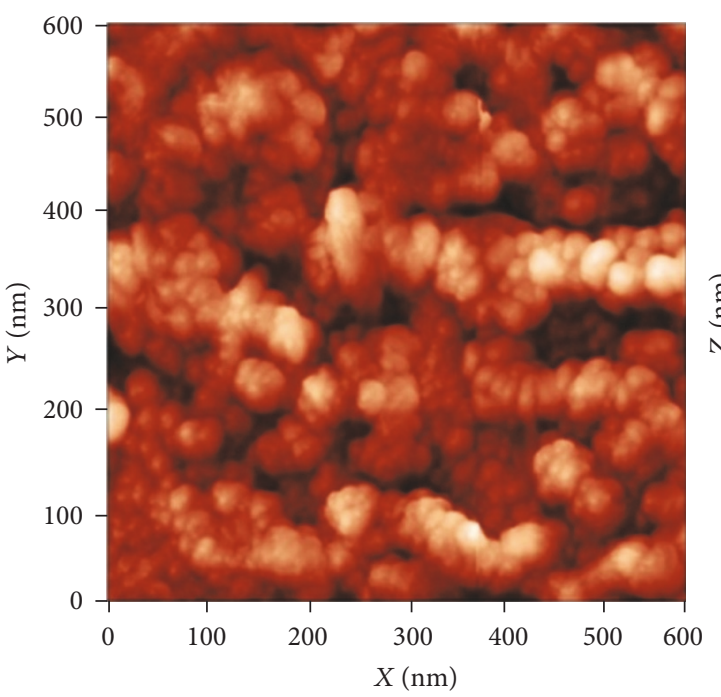

(c)
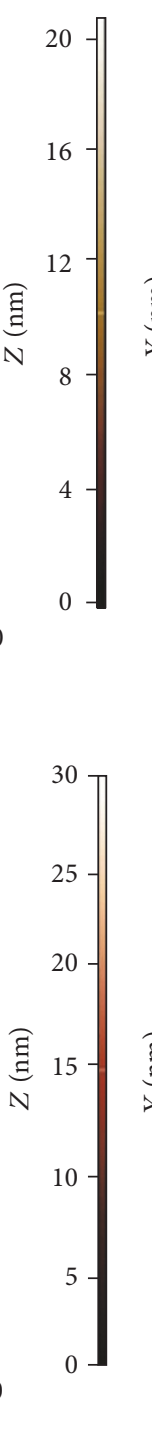

$$
\text { ] }
$$
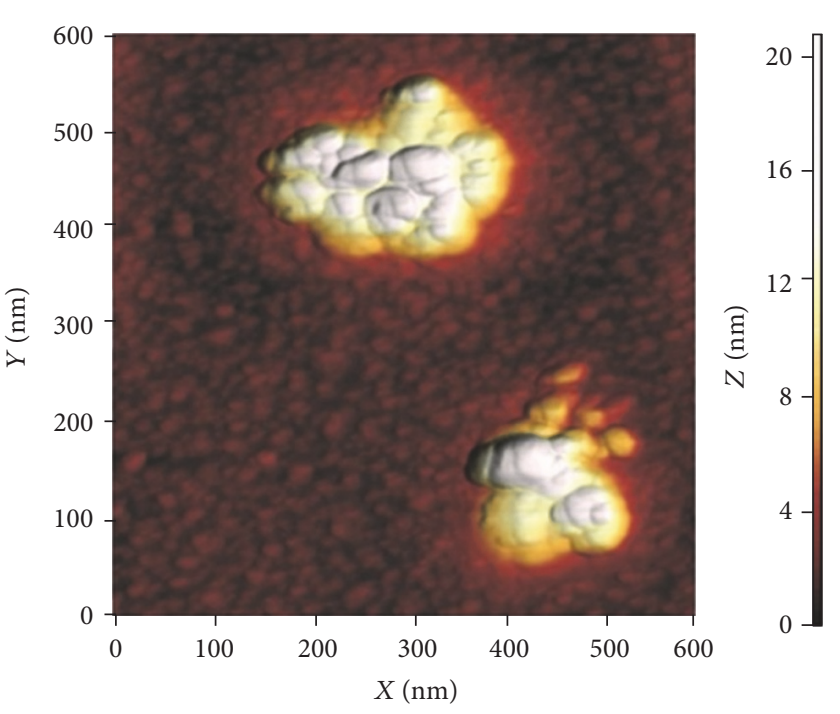

(b)
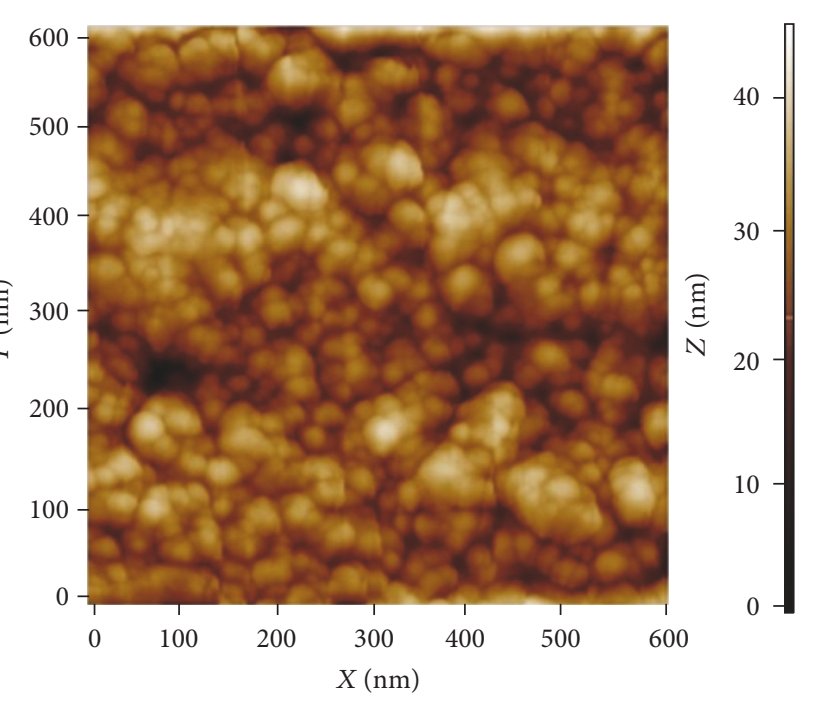

(d)

FIGURE 4: AFM images of bimetallic nanostructures deposited on a glass substrate after (a) 5 laser passages; (b) 10 passages; (c) 15 passages; and (d) 20 passages. Here, the laser power is $2.5 \mathrm{~W}$ and the scanning speed is $1.2 \mathrm{~mm} / \mathrm{s}$.

Then, with the increase in the number of laser scans, the structure is found to transform into a quasiperiodical array of nanoparticles (Figures 4(c) and 4(d)). We note that the height of the deposited film increases monotonically with the increase in the laser scan number. Finally, after $\sim 20-25$ laser scans, a granular film is formed with an average height of the relief of $\sim 52 \mathrm{~nm}$ (Figure 5(a)), which differs from the quasiperiodic nanoparticle arrays obtained for smaller number of laser passages at smaller laser speed (Figure 5(b)).

In addition to the number of laser passages, scanning speed also affects the deposited film. Figure 5 compares the morphology of the film obtained with the scanning speed of $1.2 \mathrm{~mm} / \mathrm{s}$ and $0.6 \mathrm{~mm} / \mathrm{s}$. Thus, a control over the deposition process can be achieved by varying laser scanning parameters.
A more detailed analysis of the film morphology reveals a variety of aggregate shapes. Some of them reveal typical fractal shapes shown in Figure 6. These structures are known as "fractal" aggregates, whose optical properties strongly depend on configurations and are discussed in Section 3.3.

\section{Optical Properties of the Deposited Films}

3.1. Measurement of the Film Optical Properties. In addition to the film morphology, the optical properties of the deposited nanoparticle films were examined. The film transmission was measured in the spectral range between $300 \mathrm{~nm}$ and $800 \mathrm{~nm}$ by using SF-2000 spectrophotometer (Figure 7). In particular, the obtained results demonstrate that the optical transmission spectra of the films strongly depend on their 


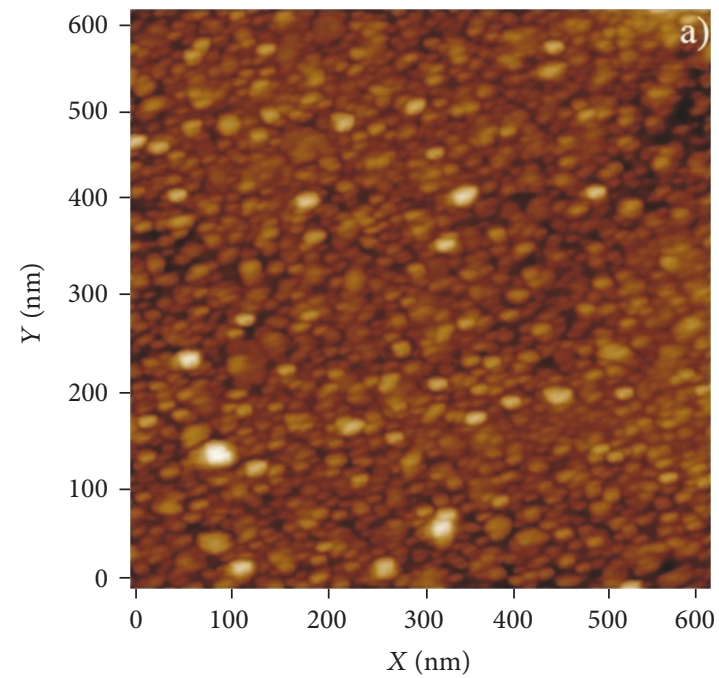

(a)
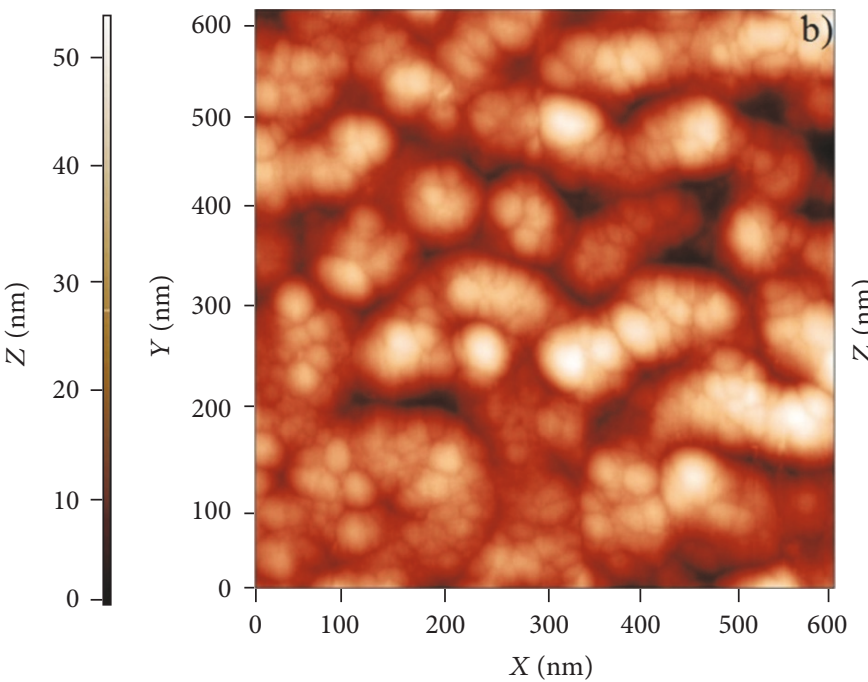

(b)

FIGURE 5: AFM images of bimetallic nanostructures deposited at the laser power of $2.5 \mathrm{~W}$ (a) after 25 passages with the scanning speed of $1.2 \mathrm{~mm} / \mathrm{s}$; and (b) after 18 passages with a speed of $0.6 \mathrm{~mm} / \mathrm{s}$.

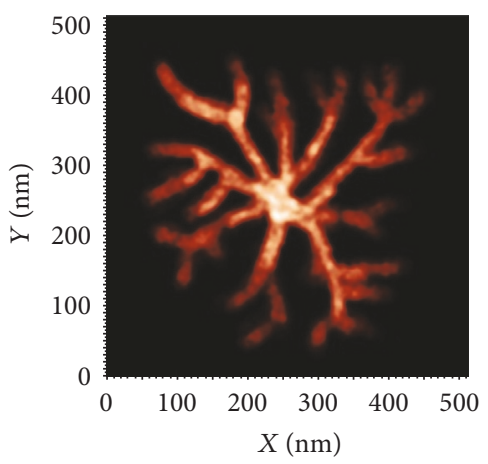

(a)
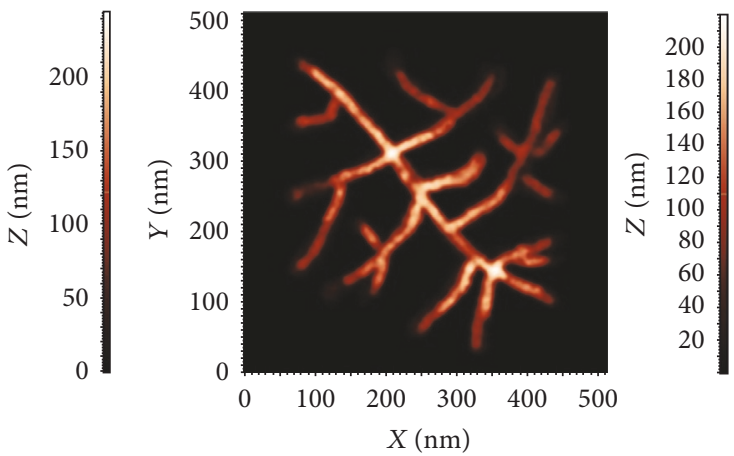

(b)
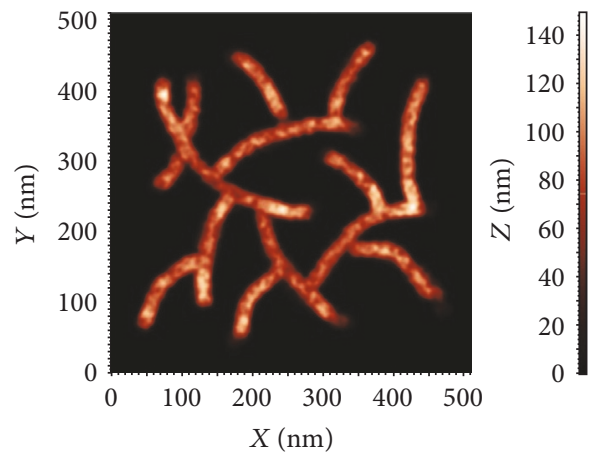

(c)

Figure 6: Three different morphologies of the obtained fractal aggregates: (a) "star" configuration; (b) “leaf” configuration; and (c) "brunches" configuration.

thickness. For a single-layer film, plasmon resonances of silver and gold nanoparticles are not detected. When the number of layers rises, however, significant changes in the transmission spectrum can be observed. Thus, a minimum is found at the plasmon resonance of gold in the transmission of all films, whereas the behavior is more complicated at the wavelength corresponding to the plasmon resonance of silver nanoparticles. This result can be explained by silver oxidation. While a single minimum is observed in the transmission spectra of thinner films, it splits for the 


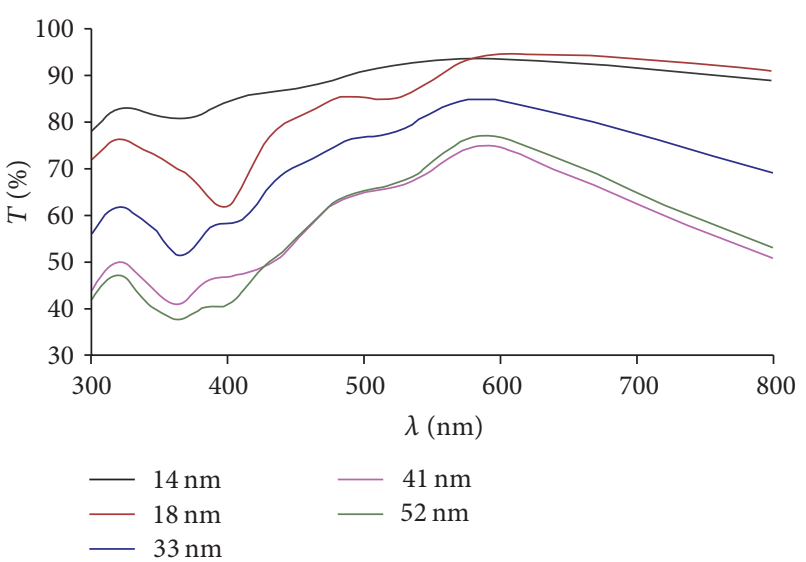

(a)

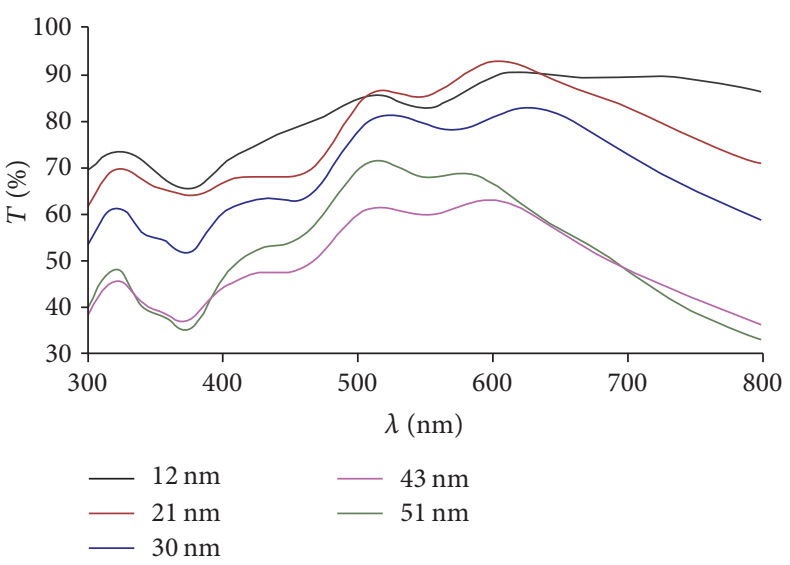

(b)

FIGURE 7: Measured transmission spectra of bimetallic films as a function of the average height.

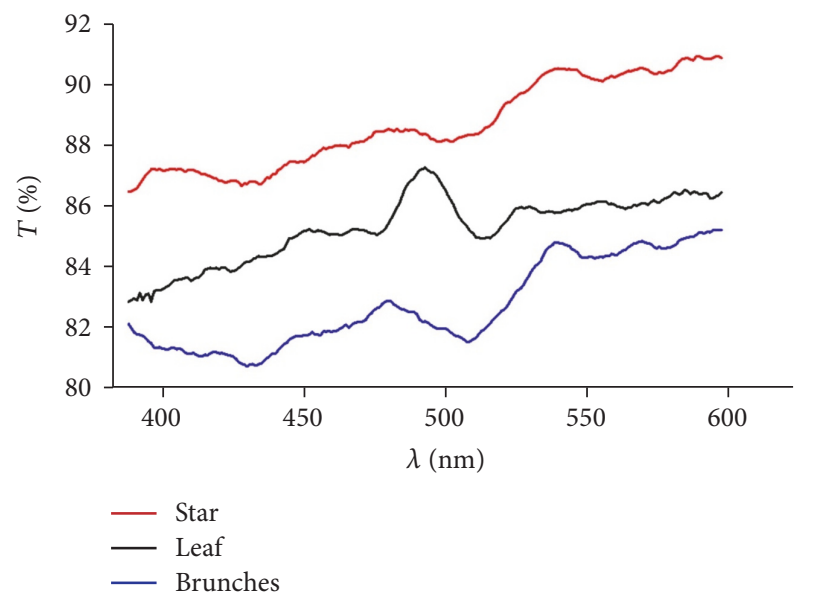

FIGURE 8: Measured transition spectra of fractal aggregate shown in Figure 4.

thicker films. The transmission of all films decays sharply at wavelengths larger than $\sim 600 \mathrm{~nm}$. This result is typical for continuous metal films with the trends becoming more pronounced for thicker films.

It should be mentioned that the transmission of a twolayer film is larger in the considered part of the spectrum than the one of a single-layer film. A similar trend is observed if one compares four- and five-layered films. For all the multilayer films, a periodic change in the transmittance is observed between 400 and $500 \mathrm{~nm}$. This result can be explained by the formation of silver-gold alloy nanoparticles in the colloids $[8-10,14,15]$.

In addition, the transmission spectra are found to strongly depend on the aggregate shape. Figure 8 illustrates this dependency for three typical fractal aggregate configurations shown in Figure 6.

3.2. FDTD Modeling of the Effects of the Particle Spacing and the Number of Layers on the Film Optical Properties. Firstly, the transmission spectra of the granular metal films

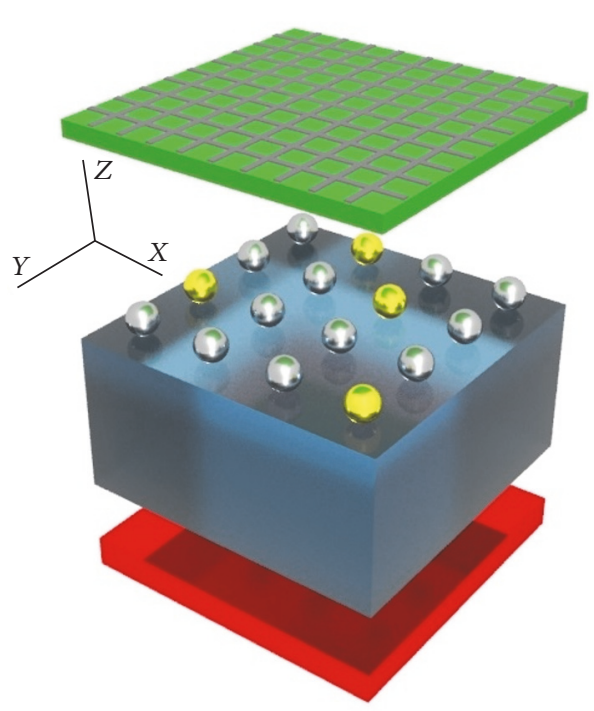

FIGURE 9: Schematics of the computational domain that consisted of a glass substrate with sixteen silver and gold nanoparticles. Below the glass substrate is a plane wave source. The detectors are located above the air region.

are calculated for different spacing between the particles and for a various number of particle layers. For this, Finite Difference Time Domain method (FDTD) is used $[8,16]$. In this case, the optical properties of metals are described by Drude-Lorentz model $[17,18]$. The model system consists of gold and silver spheres. As one can see in Figure 9, $10 \mathrm{~nm}$ diameter spheres are located at the nodes of a $6 \times 6$ square grid and are placed on the top of a substrate with refractive index of 1.5 corresponding to KV8 glass. The distance between the spheres along $X$ and $Y$ coordinates is then varied between 0 and $5 \mathrm{~nm}$. In the case of multilayer systems, metal spheres are located one above another without any gap. The nodes are filled with silver or gold spheres at random; the probability of filling a node by a silver sphere is $2 / 3$, whereas the probability of filling a node by a gold sphere is $1 / 3$. 


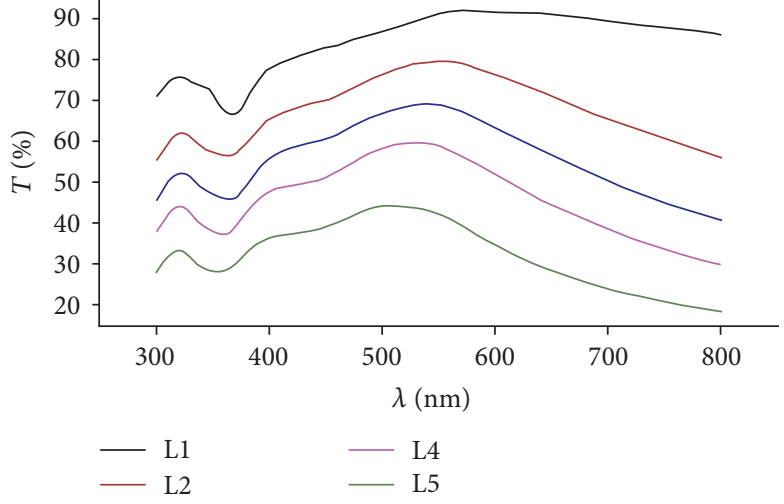

(a)

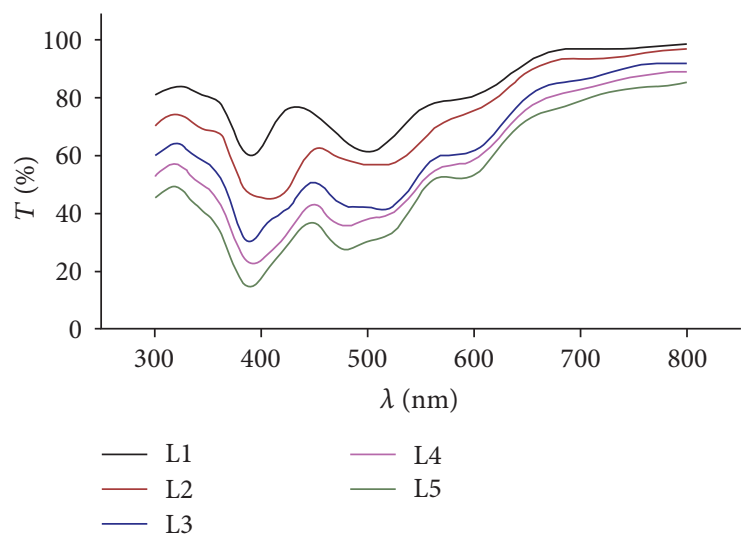

(c)

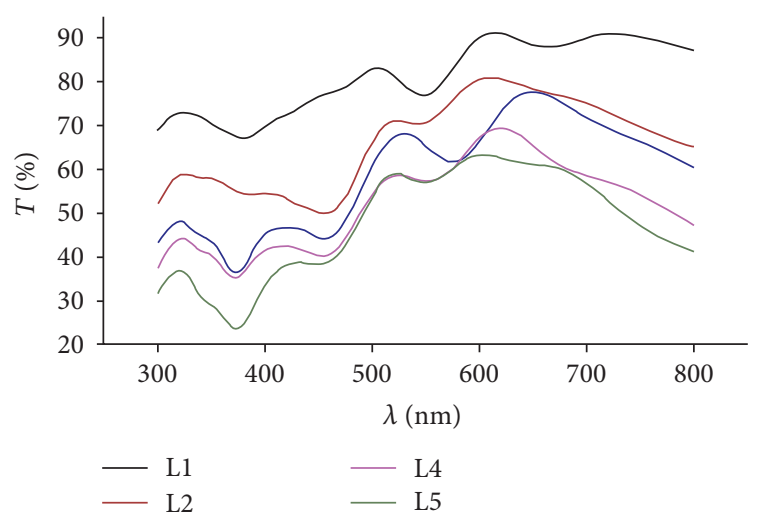

(b)

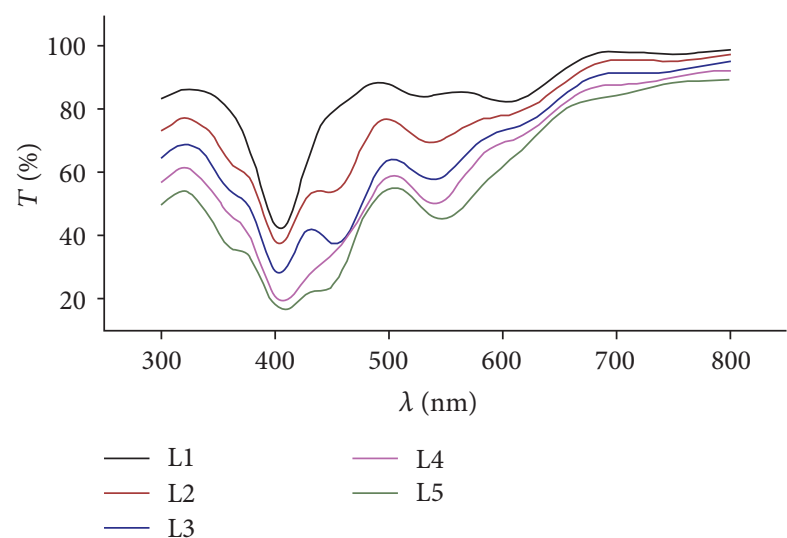

(d)

FIGURE 10: Calculated transmission spectra of the films composed of gold-silver particles with the diameter of $10 \mathrm{~nm}$ that are located on a glass substrate with different spacing between the particles: (a) $0 \mathrm{~nm}$, (b) $2 \mathrm{~nm}$, (c) $4 \mathrm{~nm}$, and (d) $6 \mathrm{~nm}$. The number of layers in the films is varied from 1 to 5 .

The top and the bottom of the simulation volume are limited by the perfectly matched layers (PML layer) with the thickness of $100 \mathrm{~nm}$, whereas the periodic boundary conditions are set at the side walls. The laser source is located below the substrate. The laser polarization is directed along the $O X$ axis, so that the source provides a plain wavefront parallel to the XOY-plane. The calculations are performed for 10 optical cycles. The transmission is defined as the ratio of the intensity in a plane coinciding with the bottom of the PML layer above the particles, to the source intensity. In all cases, the calculated spectra are quite similar. The variations obtained for different locations of gold and silver spheres do not exceed $4 \%$. Therefore, the calculation results are shown for only one particular configuration.

The obtained calculation results are presented in Figure 10 clearly demonstrating that the mean spacing strongly affects the transmission spectra of the films. Whereas the particle coupling strongly affects the spectra of closely packed nanoparticles (Figures 10(a) and 10(b)), the typical gold and silver localized plasmon resonances become more and more pronounced with the increase in the spacing (Figures 10(c) and $10(\mathrm{~d})$ ). This result is certainly due to the fact that with the increase of particle spacing, the particle coupling decreases.
Additionally, the film transmittance drops significantly with the increase in the number of layers (Figure 10), whereas the typical localized plasmon resonances of gold and silver nanoparticles can be still observed for larger spacing. On one hand, fairly no sign of the plasmon resonances is obtained in the transmission spectra of the films with small spacing. On the other hand, when the spacing becomes comparable with or overcomes the mean particle sizes, plasmon absorption can be clearly revealed both for gold and silver nanoparticle arrays.

The difference between the simulation results and the experimental values can be explained by the fact that the assumed particle arrangement can differ from the real one and because of the limited system size. We believe, however, that the main observed effects are reproduced correctly.

3.3. Effects of the Aggregate Morphology on the Film Optical Properties. In addition to the particle spacing and the number of particle layers, here we examine the effects of the aggregate morphology on the transmission spectra. For this, a series of calculations are performed (Figure 11) by using the generalized Mie theory $[19,20]$. The calculations are performed for aggregates composed of 24 particles with particle 


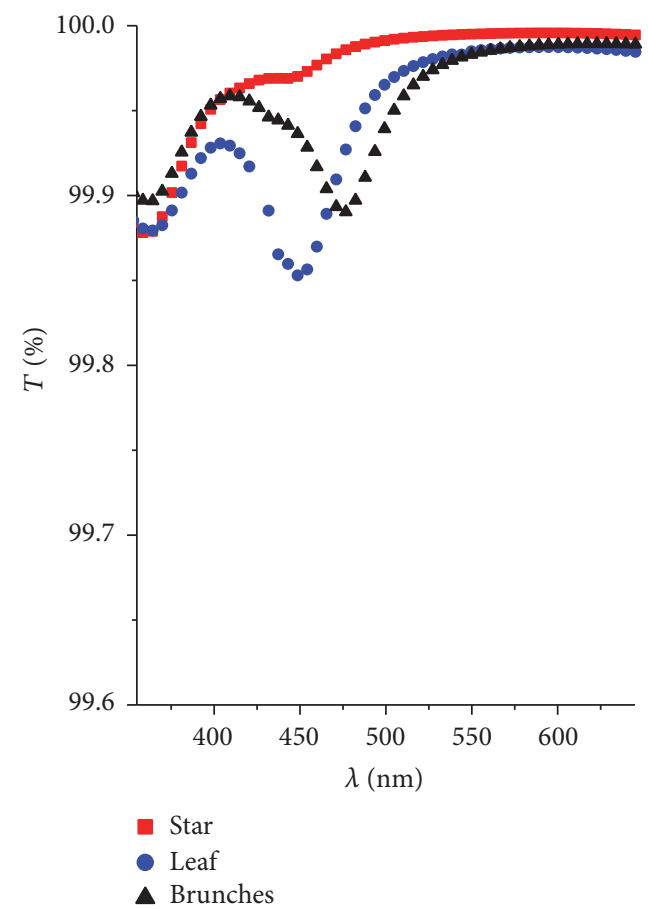

FIGURE 11: Calculated transmission spectra for three different fractal aggregate configurations shown in Figure 5.

diameter of $10 \mathrm{~nm}$ that are located in glass (sodium-calcium glass from Schott Refractive Index Database [20]). Here, we set the same ratio of gold and silver particles as in the presented experiments, and particles are arranged in structures similar to the ones shown in Figure 5. The transmittance was calculated based on the complete extinction coefficient [14].

The obtained results can be correlated with the experimentally measured spectra shown in Figure 6. In addition to the localized surface plasmon resonance peaks characteristic for single nanoparticles, mode hybridization is known to occur in nanoparticle systems. Such effects, as well as Fano resonance, are particularly important for closely packed metal nanoparticles [21]. The difference is mainly due to the limited number of particles and, hence, smaller aggregates. The obtained transmission depends on a number of additional geometry parameters, such as the length of the longest nanoparticle chain and the presence or absence of the particle in the center of the aggregate and aggregate symmetry. As a result, the measured transmitting spectra of the aggregate films are affected by the presence of different configurations. More detailed investigations of these effects are underway.

\section{Conclusions}

A scanning pulsed laser system has been used for laser deposition of metallic nanoparticles from water-based colloids on glass substrates. The possibility of the formation of nanometric thin films has been demonstrated by using gold and silver nanoparticles. In particular, bimetallic $\mathrm{Au}: \mathrm{Ag}$ thin films have been successfully deposited on a glass substrate. The obtained film morphology has been found to vary from island and fractal structures to nanoparticle arrays with different mean spacing. Our results demonstrate that the properties of the films strongly depend on their morphology.

The results of the presented electromagnetic simulations explain the effects of particle spacing and the role of film thickness. Our calculations have, furthermore, confirmed the experimentally observed difference in the transmittance spectra for several fractal aggregate configurations. The proposed study opens ways for the synthesis of island films, fractal aggregates, or surface arrays composed of gold, silver, or bimetallic nanoparticles with well-controlled optical properties.

\section{Conflicts of Interest}

The authors declare that they have no conflicts of interest.

\section{Acknowledgments}

This study was funded by the Ministry of Education and Science of the Russian Federation VISU, Project no. 16.1123.2017, by RFBR Grant no. 16-32-60067 mol_a_dk, by President Grant MK-2842.2017.2. T. A. Vartanyan acknowledges support from the Ministry of Education and Science of the Russian Federation under Program no. 3.4903.2017/VU; T. E. Itina is grateful to the GENCI CINES for computer support (Project no. A0020805015) and to the PHC KOLMOGOROV France-Russia joint project FORMALAS.

\section{References}

[1] T. V. Shahbazyan and M. I. Stockman, "Challenges and advances in computational chemistry and physics," Plasmonics: Theory and Applications, vol. 15, 2013.

[2] C. Mayer, R. Palkovits, G. Bauer, and T. Schalkhammer, "Surface enhanced resonance of metal nano clusters: a novel tool for Proteomics," Journal of Nanoparticle Research, vol. 3, no. 5, pp. 359-369, 2001.

[3] S. Bakhti, A. V. Tishchenko, X. Zambrana-Puyalto, and etal., "Fano-like resonance emerging from magnetic and electric plasmon mode coupling in small arrays of gold particles," Scientific Reports, vol. 6, no. 1, article 32061, 2016.

[4] B. N. J. Persson and A. Liebsch, "Optical properties of twodimensional systems of randomly distributed particles," Physical Review B, vol. 28, no. 8, pp. 4247-4254, 1983.

[5] K. Baba, T. Okuno, and M. Miyagi, "Silver-gold compound metal island films prepared by using a two-step evaporation method," Applied Physics Letters, vol. 62, no. 5, pp. 437-439, 1993.

[6] A. Tlahuice-Flores, "Optical properties of thiolate-protected Ag n Au25-n (SCH3)18- Clusters," Journal of Nanoparticle Research, vol. 15, no. 7, article no. 1771, 2013.

[7] K. N. Tu, "Irreversible processes of spontaneous whisker growth in bimetallic $\mathrm{Cu}-\mathrm{Sn}$ thin-film reactions," Physical Review B, vol. 49, no. 3, pp. 2030-2034, 1994.

[8] A. Antipov, S. Arakelian, T. Vartanyan, and etal., "Optical properties of multilayer bimetallic films obtained by laser deposition of colloidal particles," Optics and Spectroscopy, vol. 121, no. 5, pp. 765-768, 2016.

[9] A. O. Kucherik, S. M. Arakelyan, S. V. Kutrovskaya et al., "Laser deposition of bimetallic island films," in Proceeding of the $V$ 
International Conference of Photonics and Information Optics, Journal of Physics: Conference Series, 737.

[10] S. M. Arakelyan, V. P. Veiko, S. V. Kutrovskaya et al., "Reliable and well-controlled synthesis of noble metal nanoparticles by continuous wave laser ablation in different liquids for deposition of thin films with variable optical properties," Journal of Nanoparticle Research, vol. 18, no. 6, article 155, 2016.

[11] D. V. Abramov, A. A. Antipov, S. M. Arakelian et al., "New advantages and challenges for laser-induced nanostructured cluster materials: functional capability for experimental verification of macroscopic quantum phenomena," Laser Physics, vol. 24, no. 7, Article ID 074010, 2014.

[12] A. A. Antipov, S. M. Arakelyan, S. V. Kutrovskaya et al., "Pulse laser deposition of cluster nanostructures from colloidal singlecomponent systems," Bulletin of the Russian Academy of Sciences: Physics, vol. 76, no. 6, pp. 611-617, 2012.

[13] B. Lü, V. Elofsson, E. P. Münger, and K. Sarakinos, "Dynamic competition between island growth and coalescence in metalon-insulator deposition," Applied Physics Letters, vol. 105, no. 16, Article ID 163107, 2014.

[14] A. A. Antipov, S. M. Arakelyan, T. A. Vartanyan et al., "Optical properties of nanostructured gold-silver films formed by deposition of small colloid drops," Optics and Spectroscopy, vol. 119, no. 1, pp. 119-123, 2015.

[15] S. Arakelian, T. Vartanyan, A. Istratov et al., "Formation of quasiperiodic bimetal thin films with controlled optical and electrical properties," in Nanophotonics VI, vol. 9884 of Proceedings of SPIE, Brussels, Belgium, April 2016.

[16] S. S. Zivanovic, K. S. Yee, and K. K. Mei, "A subgridding method for the time-domain finite-difference method to solve Maxwell's equations," IEEE Transactions on Microwave Theory and Techniques, vol. 39, no. 3, pp. 471-479, 1991.

[17] D. Barchiesi and T. Grosges, "Fitting the optical constants of gold, silver, chromium, titanium, and aluminum in the visible bandwidth," Journal of Nanophotonics, vol. 8, no. 1, Article ID 083097, 2014

[18] F. Hao and P. Nordlander, "Efficient dielectric function for FDTD simulation of the optical properties of silver and gold nanoparticles," Chemical Physics Letters, vol. 446, no. 1-3, pp. 115-118, 2007.

[19] Y.-L. Xu, "Electromagnetic scattering by an aggregate of spheres: far field," Applied Optics, vol. 36, no. 36, pp. 9496-9508, 1997.

[20] Y. Xu, "Electromagnetic scattering by an aggregate of spheres: asymmetry parameter," Physics Letters A, vol. 249, no. 1-2, pp. 30-36, 1998.

[21] M. I. Tribelsky, S. Flach, A. E. Miroshnichenko, A. V. Gorbach, and Y. S. Kivshar, "Light scattering by a finite obstacle and fano resonances," Physical Review Letters, vol. 100, no. 4, Article ID 043903, pp. 1-4, 2008. 

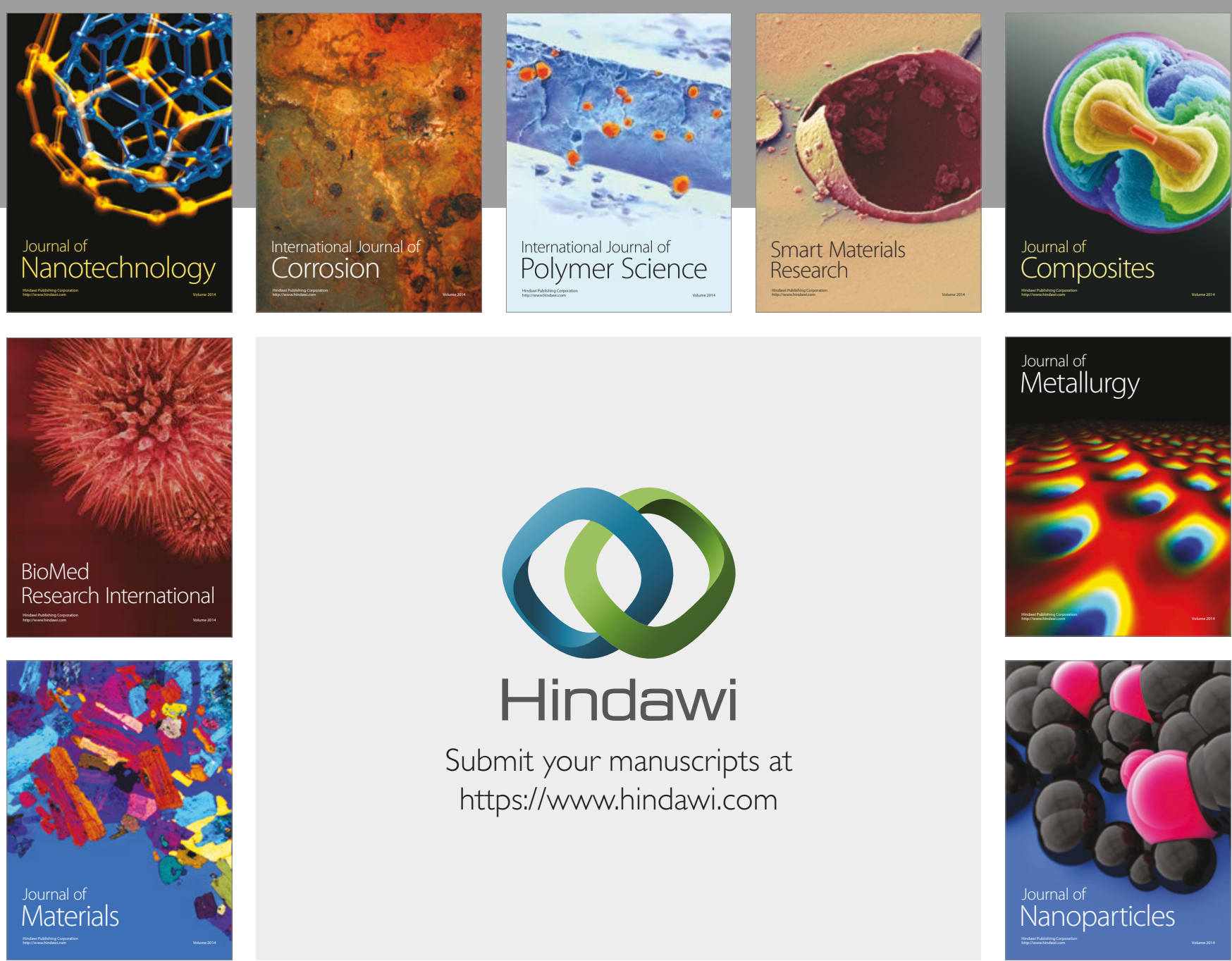

\section{Hindawi}

Submit your manuscripts at

https://www.hindawi.com
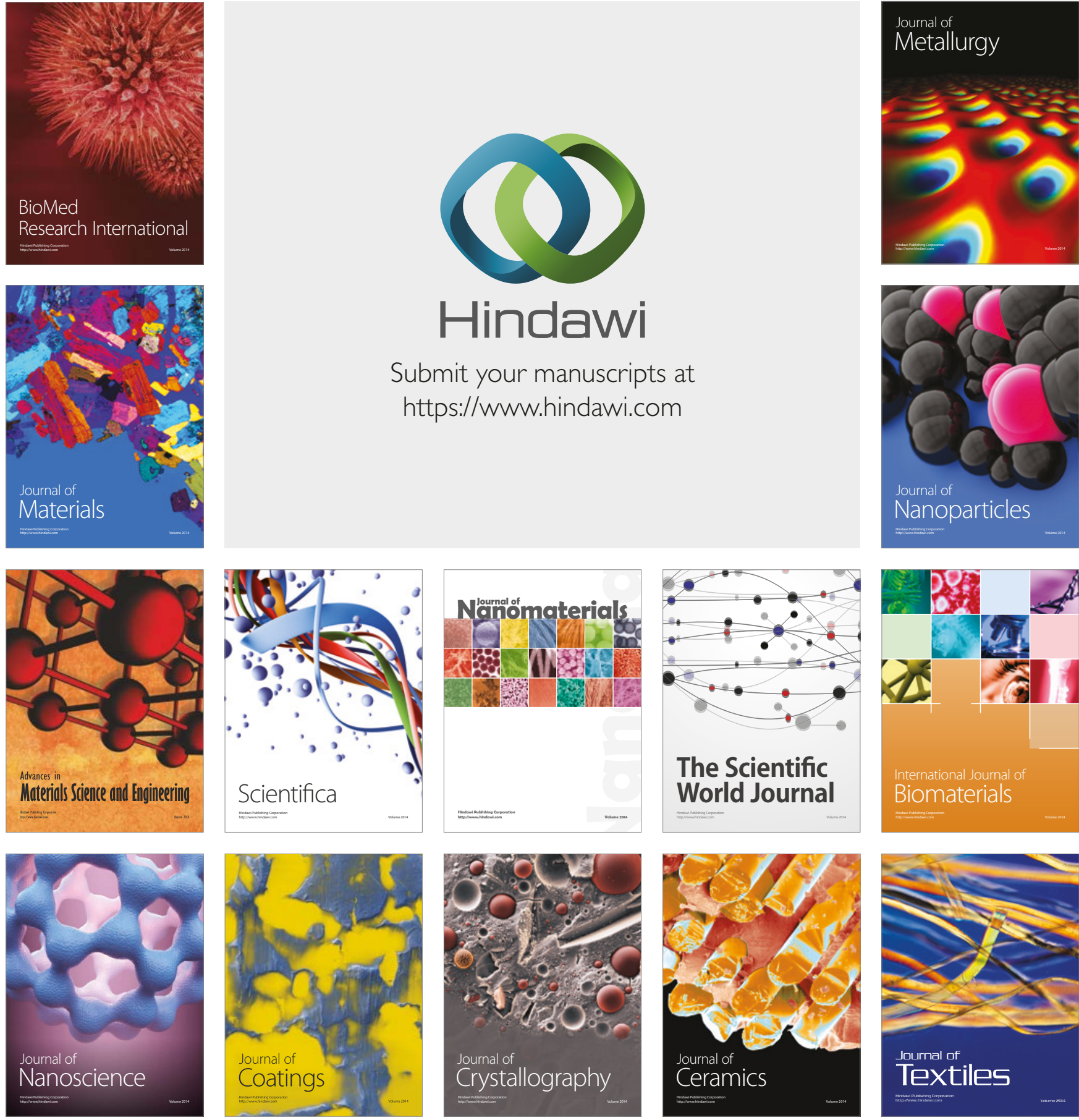

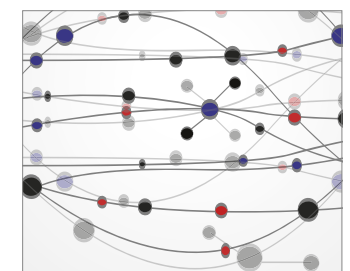

The Scientific World Journal
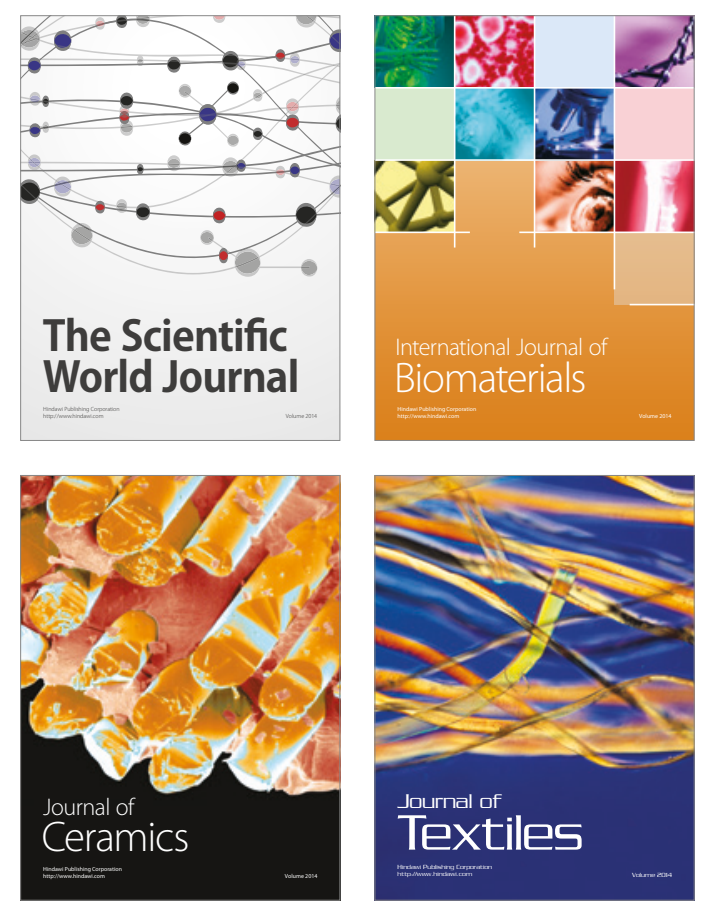